\title{
FORC Analysis of homogeneous nucleation in the two-dimensional kinetic Ising model
}

\author{
D.T. Robb* \\ School of Computational Science, Florida State University, Tallahassee, FL 32306 and \\ ERC Center for Computational Sciences, Mississippi State University, Mississippi State, MS 39762 \\ M.A. Novotny \\ Department of Physics and Astronomy and ERC Center for Computational Sciences, \\ Mississippi State University, Mississippi State, MS 39762 \\ P.A. Rikvold $\sharp$ \\ School of Computational Science, Center for Materials Research and Technology, \\ and Department of Physics, Florida State University, Tallahassee, FL 32306 and \\ National High Magnetic Field Laboratory, Tallahassee, FL 32310
}

(Dated: October 3, 2018)

\begin{abstract}
The first-order reversal curve (FORC) method is applied to the two-dimensional kinetic Ising model. For the system size and magnetic field chosen, the system reverses by the homogeneous nucleation and growth of many droplets. This makes the dynamics of reversal nearly deterministic, in contrast to the strongly disordered systems previously studied by the FORC method. Consequently, the FORC diagrams appear different from those obtained in previous studies. The KolmogorovJohnson-Mehl-Avrami (KJMA) theory of phase transformation by nucleation and growth is applied to the system. Reasonable agreement with the Monte Carlo simulations is found, and the FORC method suggests how the KJMA theory could be extended.
\end{abstract}

PACS numbers: 75.60.-d, 77.80.Dj, 64.60.Qb, 05.50.+q

\section{INTRODUCTION}

The study of hysteresis loops can produce significant dynamical information, but it reduces the often complex dynamics of magnetization reversal to only a few quantities, usually the hysteresis loop area and the coercive field. The first-order reversal curve (FORC) method was recently developed ${ }^{1}$ to extract more information from experiments on magnetic systems. It has produced interesting results, mostly in systems with strong disorder in the physics of magnetism ${ }^{2,3,4}$ and in the geosciences. ${ }^{5.6}$

\section{FORC ANALYSIS AND MONTE CARLO SIMULATION}

The FORC technique involves saturating the magnetization $M$ in a positive field $H_{0}$, decreasing the field to a series of progressively more negative return fields $H_{r}$, and then in each case increasing the field back to $H_{0}$ while recording the magnetization $m=M / M_{s}$, where $M_{s}$ is the saturation magnetization. This results in a set of curves $m\left(H_{r}, H\right)$, where $H$ represents the field as it is increased from $H_{r}$ back to $H_{0}$, as shown in Fig. 11(a).

We use FORC analysis to better understand the process of hysteresis in the two-dimensional Ising model, which is defined by the Hamiltonian

$$
\mathcal{H}=-J \sum_{\langle i, j\rangle} S_{i} S_{j}-\mu H(t) \sum_{i} S_{i} ;
$$

where the lattice spins $S_{i}= \pm 1$, the exchange constant $J$ is in units of energy, $\mu$ is the magnetic moment per site, and $H(t)$ is the applied time-dependent field. The simulation is run on an $L \times L$ square lattice with periodic boundary conditions. The first sum is taken over all nearest-neighbor pairs of spins, and the second sum over all individual spins. Hereafter we use units such that $J=\mu=k_{B}=1$. Our simulations are run at a temperature of $T=0.8 T_{c}$, and we use the Glauber dynamic with random, single-site updates for the Monte Carlo (MC) simulation 7 The field decreases linearly at the sweep rate $-\Omega$ from $H_{0}$ to $H_{r}$, and then increases linearly at the sweep rate $+\Omega$ back to $H_{0}$.

The free-energy barrier for the metastable state in the Ising system leads either to a co-existence (CE), singledroplet (SD), multi-droplet (MD), or strong-field (SF) regime for magnetization reversal $\stackrel{8}{-}$ Occurring at intermediate values of system size, field and temperature, the MD regime is arguably the most accessible to experiments. The SF regime requires fields that may be too large to generate experimentally, while the SD regime requires small systems or very low temperatures, and thus very long metastable lifetimes. To ensure that the reversal is in the MD regime, the saturation field is chosen as $H_{0}=0.545$, and the system size as $L=512$. We observed directly that at $L=512$ more than 100 droplets were involved in the reversal, and our FORC results did not change appreciably for larger $\mathrm{L}$ or larger $H_{0}$. We calculated the characteristic reversal time in a constant field $H=-H_{0}$, defined as the time to decrease from $m=1.0$ to $m=-0.8$, to be roughly 100 MCSS (MC steps per spin). We therefore chose $\Omega=2.18 \times 10^{-3} \mathrm{MCSS}^{-1}$, so that the time for the field to decrease from $H=0$ to $H=-H_{0}$ was long enough to produce full reversal. 
In Fig. 廿(a) we present the resulting family of FORCs, where each FORC is an average over $20 \mathrm{MC}$ realizations.

It is useful to generate from this family of FORCs the FORC distribution,

$$
\rho\left(H_{r}, H\right)=-\frac{1}{2} \frac{\partial^{2} m\left(H_{r}, H\right)}{\partial H_{r} \partial H} .
$$

The motivation for this definition is that when the FORCs are taken from a Preisach system, the FORC distribution reproduces the Preisach distribution $1 \frac{1}{1}$ In order to calculate the FORC distribution from our MC data, as in Ref. 1 we fit the family of curves $m\left(H_{r}, H\right)$ around each point by a second-order polynomial $g\left(H_{r}, H\right)=$ $a H_{r} H+b H_{r}^{2}+c H^{2}+d H_{r}+e H+f$, using a linear leastsquares method. The FORC distribution is most clearly displayed in a contour plot of its level curves, called a 'FORC diagram,' which we show in Fig. 22a).

There are several interesting features of the FORC results. First, a sizable part of the FORC diagram is negative. Since the FORC distribution measures how the slope $\partial m / \partial H$ varies from one FORC to the next, which can be quite different for different dynamics, this is a reasonable result. It is only in the case of the Preisach model that the FORC distribution must by definition be positive. Second, the positions of the minima of the FORCs, naively expected to be at $H=0$, gradually shift from a small negative field to a small positive field. We will provide insight into these features in section III]

\section{APPLICATION OF THE KJMA THEORY}

We briefly sketch the idea of the Kolmogorov-JohnsonMehl-Avrami (KJMA) theory. See Refs. 8 and 9 for more complete descriptions. In the present case of MD reversal, the system parameters are related as $a \ll r_{c} \ll r_{0} \ll$ $L$, where these parameters represent the lattice spacing, the critical droplet radius, the average distance before droplets meet during growth, and the system size, respectively. The critical droplet radius is the radius below which droplets of overturned spins tend strongly to shrink, and above which they tend strongly to grow.

For the MD regime, magnetization reversal can be approximated by the KJMA theory of droplet nucleation and growth, in which critical droplets are assumed to nucleate and grow with field- and temperature-dependent rates. First, the extended volume $\Phi(t)$ of the expanding droplets, uncorrected for droplet overlap, is calculated. Then the expression $\phi(t)=\exp [-\Phi(t)]$ is applied to find the volume $\phi(t)$ of overturned spins, corrected for droplet overlap. (See Ref. 9 and references cited therein.)

To apply the KJMA theory, we included the linearly varying field in the KJMA expressions, found straightforward equations for the extended volume in the region of negative fields, and then numerically integrated these equations. As in Sides et al., $\frac{9}{\underline{9}}$ we approximated the behavior of the system in positive fields as a combination of a shrinking negative metastable region, whose volume is estimated as the time reversal of the calculated growth in negative fields, and nucleation of a positive stable phase within this region, estimated from the calculated nucleation in negative fields. Because the calculated growth in negative fields extends only to $H=H_{r}$, this approximation can continue only up to $H=\left|H_{r}\right|$. However, the system at $H=\left|H_{r}\right|$, where positive droplets are growing, can be approximated by a similar (but oppositely magnetized) state of the system, obtained from the full hysteresis loop. We found the point in the calculation of the full hysteresis loop where the magnetization is equal in magnitude to, but opposite in sign from, the magnetization at $H=\left|H_{r}\right|$. Beginning with the results of the KJMA integration at this point, we continued the integration from $H=\left|H_{r}\right|$ to $H=H_{0}$.

In comparing the calculated results to the MC simulation data, we note first that the approximation of treating the final portion of each FORC loop using results from the KJMA simulation in negative fields works well, deviating only in small kinks (almost invisible on the scale used here) which develop in the first FORCs in Fig. 1(b). This suggests that the behavior of the remaining positive metastable phase in negative field values is similar to the behavior of the remaining negative metastable phase in the last stage of the FORCs. The first FORCs are least similar, probably because they consist of several well-defined shrinking droplets, rather than an irregularly spaced and shaped volume. It is also interesting that the magnetization decreases from saturation much sooner in the MC family of FORCs, due to the presence of subcritical droplets which are neglected in the KJMA treatment. This causes the values of the FORC distribution to be larger for the KJMA case, since the slopes of the FORCs must be higher.

We produced animations of the MC simulation in order to better understand the dynamics. In Fig. 1(a), the FORCs beginning in the range $-0.2>H_{r}>-0.25$ have their minima at $H<0$ because droplets that are just above the critical radius become subcritical and shrink as the field increases back toward $H=0$. The FORCs beginning in the range $-0.25>H_{r}>-0.32$ have their minima at values $H>0$ for a similar reason. These minima occur at $m<0$, where the magnetization can be viewed as consisting of positive droplets in a negative background. In the subsequent small positive fields, the growth of the larger positive droplets is accompanied by the shrinking of the smaller, subcritical positive droplets. The entire shift of the minima is reflected in the presence of values $\rho<0$ of the FORC distribution in the region $\left(-0.30<H_{r}<-0.25,-0.05<H<0.05\right)$ in Fig. 22(a).

In areas of the FORCs in which a full reversal occurred, there is a loss of memory in the MC simulation, resulting in a value $\rho \approx 0$ for these areas in Fig. 22(a). We also studied the families of FORCs and FORC diagrams for the same system with $\Omega=2.18 \times 10^{-4} \mathrm{MCSS}^{-1}$ (not shown). We found the form of the MC and KJMA results were quite similar to the $\Omega=2.18 \times 10^{-3} \mathrm{MCSS}^{-1}$ case, except that the coercive field was about half as large. 

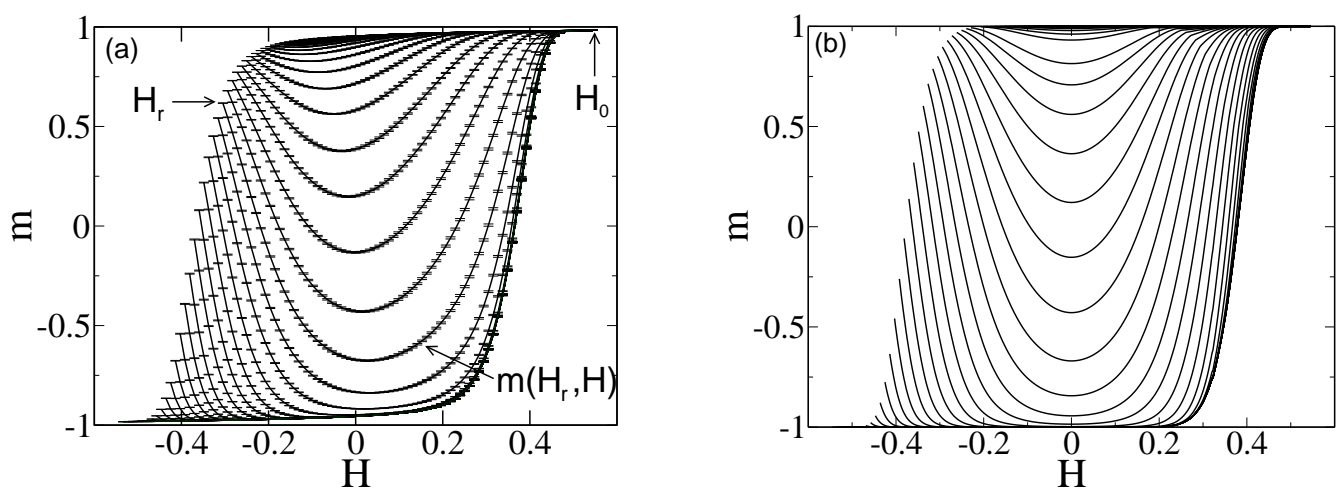

FIG. 1: Family of FORCs for $L=512$ kinetic Ising model at temperature $T=0.8 T_{c}$ in a linearly varying field with sweep rate $\Omega=2.18 \times 10^{-3} \mathrm{MCSS}^{-1}$. (a) Data from MC simulation. (b) Calculation from application of KJMA theory.
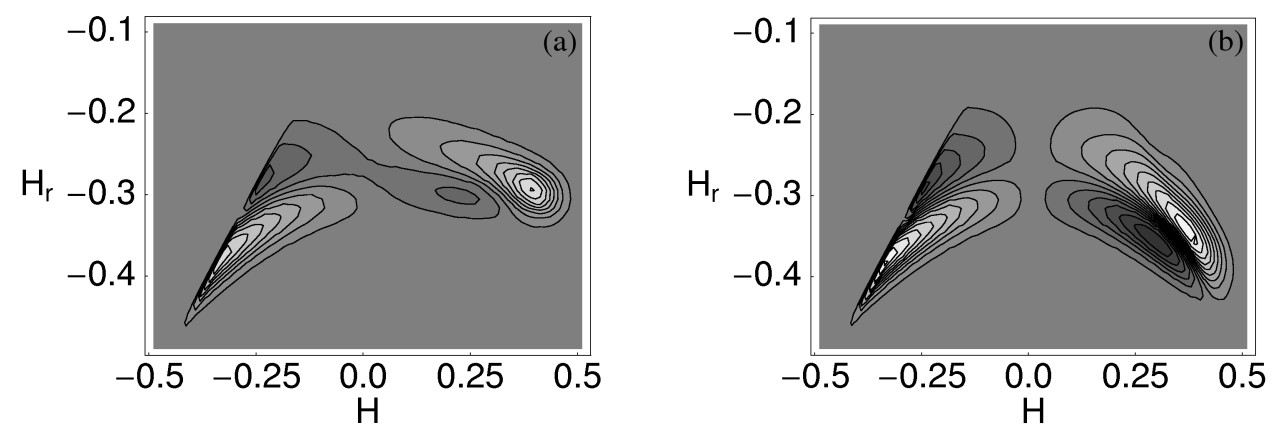

FIG. 2: FORC diagrams for the system described in Fig. 1 The magnitudes in the contour plot range from $\rho=-400$ (black) to $\rho=0$ (large gray area) to $\rho=+400$ (white). (a) Calculated from MC simulation data. (b) Calculated with KJMA theory.

\section{CONCLUSION}

In conclusion, we have applied FORC analysis to the two-dimensional kinetic Ising model, finding reasonable agreement between the predictions of KJMA theory and the results of MC simulation. The FORC analysis offers insight into the discrepancies between the KJMA and MC results, which we have identified as due to the effects of subcritical droplets and loss of memory.

In future work, we hope to extend the KJMA theory to include these effects and to make predictions with the KJMA theory for longer time scales, where MC simula- tion is not practical. Although the simulation results for $\Omega=2.18 \times 10^{-3} \mathrm{MCSS}^{-1}$ and $\Omega=2.18 \times 10^{-4} \mathrm{MCSS}^{-1}$ were very similar, the long time scales of physical systems may produce different and physically relevant insights into the dynamics of the homogeneous reversal process.

We thank H.G. Katzgraber and C.R. Pike for useful comments. This work was supported by NSF Grant No. DMR-0120310, by the ERC Center for Computational Sciences at Mississippi State University, and by the School of Computational Science at Florida State University.
* Electronic address: robb@csit.fsu.edu

$\dagger$ Electronic address: novotny@erc.msstate.edu

¥ Electronic address: rikvold@csit.fsu.edu

1 C. Pike, A. Roberts, and K. Verosub, J. Appl. Phys. 85, 6660 (1999).

2 P. G. Bercoff, M. I. Oliva, E. Broclone, and H. R. Bertorello, Physica B 320, 291 (2002).

${ }^{3}$ H. G. Katzgraber, F. Pàzmàndi, C. R. Pike, K. Liu, R. T.
Scalettar, K. L. Verosub, and G. T. Zimànyi, Phys. Rev. Lett. 89, 257202 (2002).

${ }^{4}$ C. R. Pike, Phys. Rev. B 68, 104424 (2003).

5 A. Roberts, C. R. Pike, and K. Verosub, J. Geophys. Res. 105, 28461 (2001).

6 A. R. Muxworthy and D. J. Dunlop, Planetary Science Letters 203, 369 (2002).

7 D. P. Landau and K. Binder, A Guide to Monte Carlo Sim- 
ulations in Statistical Physics (Cambridge University Press, Cambridge, UK, 2000), pp. 68-73.

8 P. A. Rikvold, H. Tomita, S. Miyashita, and S. W. Sides, Phys. Rev. E 49, 5080 (1994).
9 S. W. Sides, P. A. Rikvold, and M. A. Novotny, Phys. Rev. E 59, 2710 (1999). 